\title{
Clinical significance of prognostic inflammation-based and/or nutritional markers in patients with stage III gastric cancer
}

Takahiro Toyokawa ${ }^{1 *}$ (D, Kazuya Muguruma ${ }^{1}$, Mami Yoshii ${ }^{1}$, Tatsuro Tamura ${ }^{1}$, Katsunobu Sakurai ${ }^{2}$, Naoshi Kubo $^{2}$, Hiroaki Tanaka', Shigeru Lee ${ }^{1}$, Masakazu Yashiro ${ }^{1}$ and Masaichi Ohira ${ }^{1}$

\begin{abstract}
Background: Although many studies have identified several inflammation-based and/or nutritional markers with prognostic value for patients with various types of cancer, the optimal markers and cut-off values for these markers remain obscure. Therefore, this retrospective study aimed to identify optimal markers and their cutoffs.

Methods: We compared prognostic values among established preoperative inflammation-based and/or nutritional markers in 225 patients who underwent R0 resection for stage III gastric cancer. Inflammationbased and/or nutritional markers comprised C-reactive protein to albumin ratio (CAR), neutrophil to lymphocyte ratio (NLR), platelet to lymphocyte ratio (PLR), prognostic nutritional index (PNI), Glasgow prognostic score (GPS), and prognostic index (PI). Time-dependent receiver operating characteristic curves were analyzed to assess predictive ability and to determine the optimal cut-off values. Prognostic factors predicting overall survival (OS) and cancer specific survival (CSS) were analyzed using Cox proportional hazards models.

Results: Multivariate analyses revealed that CAR and PLR cut-off values of 0.47 and 172, respectively, were independent prognostic factors for overall survival (OS) $(\mathrm{HR}, 2.257 ; 95 \% \mathrm{Cl}, 1.180-4.319 ; p=0.014$ and $\mathrm{HR}$, $1.478 ; 95 \% \mathrm{Cl}, 1.025-2.133 ; p=0.037$, respectively) and cancer-specific survival (CSS) (HR, $2.771 ; 95 \% \mathrm{Cl}, 1.398-$ 5.493; $p=0.004$ and $\mathrm{HR}, 1.552 ; 95 \% \mathrm{Cl}, 1.029-2.341 ; p=0.036$, respectively). These results were different from those we previously reported in patients with stage $I$.

Conclusions: Among inflammation-based and/or nutritional markers, CAR and PLR were independent prognostic factors of OS and CSS in patients with stage III gastric cancer. The optimal markers and their cutoff values should be determined in specific populations.
\end{abstract}

Keywords: Gastric cancer, C-reactive protein to albumin ratio, Platelet to lymphocyte ratio, Nutrition, Inflammation

\footnotetext{
* Correspondence: t-toyokawa@med.osaka-cu.ac.jp

'Department of Gastroenterological Surgery, Osaka City University Graduate School of Medicine, 1-4-3 Asahimachi, Abeno-ku, Osaka 545-8585, Japan

Full list of author information is available at the end of the article
}

(c) The Author(s). 2020 Open Access This article is licensed under a Creative Commons Attribution 4.0 International License, which permits use, sharing, adaptation, distribution and reproduction in any medium or format, as long as you give appropriate credit to the original author(s) and the source, provide a link to the Creative Commons licence, and indicate if changes were made. The images or other third party material in this article are included in the article's Creative Commons licence, unless indicated otherwise in a credit line to the material. If material is not included in the article's Creative Commons licence and your intended use is not permitted by statutory regulation or exceeds the permitted use, you will need to obtain permission directly from the copyright holder. To view a copy of this licence, visit http://creativecommons.org/licenses/by/4.0/ The Creative Commons Public Domain Dedication waiver (http://creativecommons.org/publicdomain/zero/1.0/) applies to the data made available in this article, unless otherwise stated in a credit line to the data. 


\section{Background}

Gastric cancer is the second leading cause of cancerrelated death worldwide [1]. Advances in diagnosis and treatment modalities have led to favorable outcomes for early-stage gastric cancer, but the postoperative survival of patients with advanced gastric cancer remains poor. Stage III gastric cancer accounts for approximately 15\% of gastric cancers, and the reported 5-year overall survival (OS) rate in Japan is $34.8-53.6 \%$ [2]. At present, TNM classification is the most generally accepted predictor of long-term outcomes and for selecting adjuvant therapies for gastric cancer in clinical practice. However, clinical outcomes vary even within the same stage because other factors influence outcomes. Therefore, other biomarkers should be identified to predict individual outcomes more precisely and to develop individual treatment strategies for patients with gastric cancer.

Many studies have indicated that not only tumorrelated factors, but also patient-related factors such as systemic inflammation and nutritional status, are involved in the prognosis of patients with cancer. Several inflammation-based and/or nutritional markers have recently been developed and preoperative markers, such as C-reactive protein to albumin ratio (CAR) [3, 4], neutrophils to lymphocyte ratio (NLR) $[5,6]$, platelets to lymphocyte ratio (PLR) [7, 8], as well as prognostic nutritional index (PNI) [9, 10], Glasgow prognostic score (GPS) [11, 12] and prognostic index (PI) [13, 14] are prognostic for various cancers. These markers are promising as clinical prognostic predictors of cancer because they are inexpensive and simple to estimate. On the other hand, the optimal markers and their cut-off value remain debatable and this could cause problems with clinical applications of these markers. We considered that these variations were attributable to different study populations. Therefore, optimal markers and their cutoff values should be determined based on a specific population such as a single tumor stage of each type of cancer to minimize discrepancies. We recently described optimal markers and their cut-off values for patients with stage II gastric cancer [15]. Here, we aimed to determine the prognostic impact of preoperative inflammation-based and nutritional markers in patients with stage III gastric cancer, and to verify whether it differs between stage II and stage III.

\section{Methods}

We retrospectively reviewed clinical data from consecutive patients with gastric cancer who underwent R0 resection at Osaka City University Hospital (Osaka, Japan) between January 1997 and December 2012. All cancers were histopathologically confirmed as stage III gastric adenocarcinoma. Fourteen patients with concomitant malignancies, 13 patients who underwent neoadjuvant chemotherapy, 15 patients with incomplete preoperative laboratory data, and 3 patients who died of postoperative complications were excluded. Ultimately, this study included 225 patients. The Ethics Committee at our institution approved this retrospective study of clinical data study, which was conducted in according with the principles of the Declaration of Helsinki.

Blood samples were routinely obtained within 1 week before surgery. The CAR was calculated by dividing the serum C-reactive protein $(\mathrm{CRP})$ value $(\mathrm{mg} / \mathrm{dL})$ by the serum albumin value $(\mathrm{g} / \mathrm{dL})$. The NLR was calculated as the number of neutrophils divided by the number of lymphocytes. The PLR was calculated as the number of platelets divided by the number of lymphocytes. The PNI was calculated as $10 \times$ serum albumin value $(\mathrm{g} / \mathrm{dL})+$ $0.005 \times$ lymphocytes $\left(/ \mathrm{mm}^{3}\right)$. The GPS was constructed from CRP and albumin values as follows. We allocated GPS of 2, 1, and 0 to patients with both elevated CRP (> $1.0 \mathrm{mg} / \mathrm{dL})$ and hypoalbuminemia $(<3.5 \mathrm{~g} / \mathrm{dL})$, elevated CRP or hypoalbuminemia, and neither of these abnormalities, respectively. The PI constructed from the number of white blood cells (WBC) and CRP values are described as P2, P1, or P0 according to elevated CRP (> $1.0 \mathrm{mg} / \mathrm{dL})$ and elevated WBC $\left(<11 \times 10^{9} / \mathrm{L}\right)$, elevated CRP or elevated WBC, and neither of these abnormalities, respectively.

We evaluated the clinical variables of age, sex, body mass index (BMI), Eastern Cooperative Oncology Group (ECOG) performance status (PS), tumor location, macroscopic type, surgical procedure, lymph node dissection, histology, lymphatic invasion, venous invasion, TNM sub-stage, tumor size and adjuvant chemotherapy. Tumor stage was determined according to the third English edition of the Japanese classification of gastric carcinoma [16]. The median values served as the cut-off values for age, BMI, and tumor size. Time-dependent receiver operating characteristic (ROC) curves of 5-year $\mathrm{OS}$ as the endpoint were calculated to evaluate the predictive ability of CAR, NLR, PLR, and PNI, and maximal Youden indices were estimated to determine the cut-off values for these markers. All patients were classified as having high and low values according to these cut-off values.

Surgical procedures were determined according to tumor size, location, and the status of resection margins. In principle, adjuvant chemotherapy with oral fluoropyrimidines (5-FU, uracil-tegafur (UFT), 5'DFUR, or S-1) was administered to patients with good general condition who provided written, informed consent. The patients were followed every 4 months for the first 2 years, every 6 months for the next 3 years, and annually thereafter. Follow-up included physical examinations, routine blood tests, measurements of tumor markers, and enhanced abdominal CT scans. These were also 
implemented when recurrence was suspected. We contacted patients, family members, or their referring physicians to obtain appropriate follow-up data if patients had not presented for follow-up.

\section{Statistical analysis}

Data were statistically analyzed using SPSS software (SPSS, Inc., Chicago, IL, USA), except for timedependent ROC curves, which were analyzed using Rproject Software, version 3.3.0. Overall survival and cancer-specific survival (CSS) rates were calculated from the date of the last surgical procedure until the date of the most recent follow-up or death, and to the date of most recent follow-up or death due to gastric cancer, respectively. These survival rates were calculated using the Kaplan-Meier method, and differences between curves were evaluated by log-rank tests Prognostic factors were investigated using Cox proportional hazards models, and hazard ratios (HRs) and 95\% confidence intervals (CIs) were calculated. Separate multivariate analyses were performed to compare the prognostic values of individual inflammation-based and/or nutritional markers with $p<0.1$ in univariate analyses because estimations of these variables overlapped. Values of $p<0.05$ were considered to be statistically significant.

\section{Results}

Predictive ability and cut-off values of inflammationbased and/or nutritional markers

According to the results of time-dependent ROC analyses, we determined the cut-off values of 0.47 for CAR, 1.90 for NLR, 172 for PLR, 45.6 for PNI, 0 for GPS, and 0 for PI, respectively. When the patients were classified into two groups based on these cut-off values, the areas under the curve (AUC) for CAR, NLR, PLR, PNI, GPS, and PI were 0.534, 0.584, 0.558, 0.575, 0.536, and 0.524, respectively (Fig. 1). We classified 211 (93.8\%) and 14 (6.2\%) patients as having low and high CAR, respectively, $99(44.0 \%)$ and $126(56.0 \%)$ patients as having low and high NLR, respectively, 141 (62.7\%) and 84 (37.3\%) patients as having low and high PLR, respectively, and $90(40.0 \%)$ and $135(60.0 \%)$ patients as having low and high PNI, respectively.
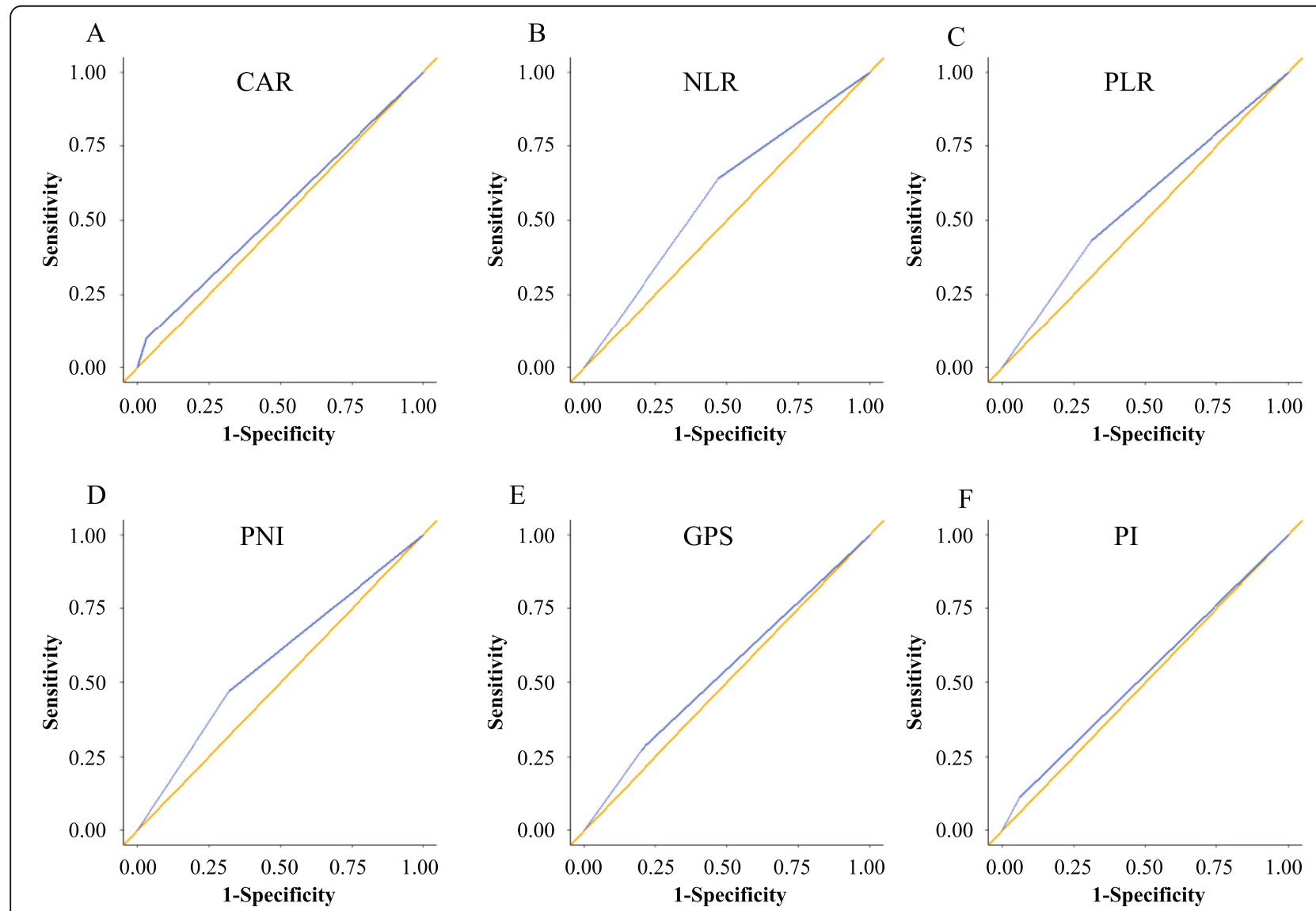

Fig. 1 Time-dependent ROC curves of 5-year OS as the endpoint for CAR (a), NLR (b), PLR (c), GPS (d)), PNI (e), and PI (f) 
Table 1 Univariate analyses of prognostic factors for OS of stage III gastric cancer

\begin{tabular}{|c|c|c|c|c|c|}
\hline \multirow[t]{2}{*}{ Variables } & \multirow{2}{*}{$\begin{array}{l}5- \\
\text { year } \\
\text { OS } \\
(\%)\end{array}$} & \multicolumn{2}{|c|}{ No. of patients } & \multicolumn{2}{|l|}{ Univariate } \\
\hline & & $n$ & $\%$ & $\mathrm{HR}(95 \% \mathrm{Cl})$ & $p$ value \\
\hline Total & 48.7 & 225 & 100 & & \\
\hline \multicolumn{6}{|l|}{ Age (years) } \\
\hline$\leq 68$ & 54.0 & 117 & 52.0 & 1 & \\
\hline$>68$ & 42.8 & 108 & 48.0 & $1.643(1.164-2.319)$ & 0.005 \\
\hline \multicolumn{6}{|l|}{ Sex } \\
\hline Male & 47.3 & 147 & 65.3 & 1 & \\
\hline Female & 51.2 & 78 & 34.7 & $1.188(0.823-1.715)$ & 0.357 \\
\hline \multicolumn{6}{|l|}{$\mathrm{BMI}\left(\mathrm{kg} / \mathrm{m}^{2}\right)$} \\
\hline Low $(\leq 21.9)$ & 46.7 & 114 & 50.7 & 1 & \\
\hline High (> 21.9) & 50.6 & 111 & 49.3 & $0.950(0.674-1.339)$ & 0.771 \\
\hline \multicolumn{6}{|l|}{ Performance status } \\
\hline 0 & 52.1 & 167 & 74.2 & 1 & \\
\hline $1-3$ & 39.1 & 58 & 25.8 & $1.606(1.109-2.325)$ & 0.012 \\
\hline \multicolumn{6}{|l|}{ Location } \\
\hline Upper/Middle/Lower & 50.7 & 209 & 92.9 & 1 & \\
\hline Whole & 20.6 & 16 & 7.1 & $1.334(1.092-1.630)$ & 0.005 \\
\hline \multicolumn{6}{|l|}{ Macroscopic type } \\
\hline Type 0/1/2 & 56.9 & 61 & 27.1 & 1 & \\
\hline Type 3/4/5 & 45.6 & 164 & 72.9 & $1.145(0.774-1.693)$ & 0.497 \\
\hline \multicolumn{6}{|l|}{ Operative procedure } \\
\hline Distal gastrectomy & 59.7 & 117 & 52.0 & 1 & \\
\hline Total gastrectomy & 36.9 & 108 & 48.0 & $1.835(1.295-2.600)$ & 0.001 \\
\hline \multicolumn{6}{|l|}{ Lymph node dissection } \\
\hline D1 & 49.8 & 32 & 14.2 & 1 & \\
\hline D2 & 48.6 & 193 & 85.8 & $0.911(0.553-1.500)$ & 0.713 \\
\hline \multicolumn{6}{|l|}{ Histology } \\
\hline Differentiated & 54.0 & 97 & 43.1 & 1 & \\
\hline Undifferentiated & 44.4 & 128 & 56.9 & $1.198(0.846-1.697)$ & 0.308 \\
\hline \multicolumn{6}{|l|}{ Lymphatic invasion } \\
\hline Absent & 57.9 & 19 & 8.4 & 1 & \\
\hline Present & 47.8 & 206 & 91.6 & $1.174(0.633-2.177)$ & 0.611 \\
\hline \multicolumn{6}{|l|}{ Venous invasion } \\
\hline Absent & 50.3 & 156 & 69.3 & 1 & \\
\hline Present & 44.8 & 69 & 30.7 & $1.193(0.830-1.716)$ & 0.340 \\
\hline \multicolumn{6}{|l|}{ TNM sub-stage } \\
\hline$\| I \mid A$ & 62.2 & 80 & 35.6 & 1 & 0.011 \\
\hline$\| \mathrm{IIB}$ & 45.4 & 72 & 32.0 & $1.579(1.030-2.420)$ & 0.036 \\
\hline$\| I C$ & 36.2 & 73 & 32.4 & $1.908(1.242-2.931)$ & 0.003 \\
\hline \multicolumn{6}{|l|}{ Tumor size (mm) } \\
\hline$\leq 60$ & 55.9 & 115 & 51.1 & 1 & \\
\hline$>60$ & 40.9 & 110 & 48.9 & $1.534(1.086-2.166)$ & 0.015 \\
\hline
\end{tabular}

Adjuvant chemotherapy 
Table 1 Univariate analyses of prognostic factors for OS of stage III gastric cancer (Continued)

\begin{tabular}{|c|c|c|c|c|c|}
\hline \multirow[t]{2}{*}{ Variables } & \multirow{2}{*}{$\begin{array}{l}5- \\
\text { year } \\
\text { OS } \\
(\%)\end{array}$} & \multicolumn{2}{|c|}{ No. of patients } & \multicolumn{2}{|l|}{ Univariate } \\
\hline & & $n$ & $\%$ & HR $(95 \% \mathrm{Cl})$ & $p$ value \\
\hline Absent & 40.1 & 41 & 18.2 & 1 & \\
\hline Present & 50.6 & 184 & 81.8 & $0.575(0.382-0.865)$ & 0.008 \\
\hline \multicolumn{6}{|l|}{ CAR } \\
\hline Low $(\leq 0.47)$ & 50.6 & 211 & 93.8 & 1 & \\
\hline High (> 0.47) & 21.4 & 14 & 6.2 & $2.844(1.561-5.181)$ & 0.001 \\
\hline \multicolumn{6}{|l|}{ NLR } \\
\hline Low $(\leq 1.90)$ & 58.0 & 99 & 44.0 & 1 & \\
\hline High (> 1.90) & 41.2 & 126 & 56.0 & $1.400(0.986-1.988)$ & 0.060 \\
\hline \multicolumn{6}{|l|}{ PLR } \\
\hline Low $(\leq 172)$ & 53.2 & 141 & 62.7 & 1 & \\
\hline High (> 172) & 40.9 & 84 & 37.3 & 1.376 (0.970-1.953) & 0.074 \\
\hline \multicolumn{6}{|l|}{ PNI } \\
\hline Low $(\leq 45.6)$ & 39.0 & 90 & 40.0 & 1 & \\
\hline High (> 45.6) & 54.9 & 135 & 60.0 & $0.730(0.517-1.031)$ & 0.074 \\
\hline \multicolumn{6}{|l|}{ GPS } \\
\hline 0 & 51.1 & 171 & 76.0 & 1 & \\
\hline $1 / 2$ & 41.1 & 54 & 24.0 & $1.463(0.992-2.156)$ & 0.055 \\
\hline \multicolumn{6}{|l|}{ PI } \\
\hline 0 & 50.1 & 205 & 91.1 & 1 & \\
\hline $1 / 2$ & 35.0 & 20 & 8.9 & $1.592(0.914-2.773)$ & 0.101 \\
\hline
\end{tabular}

BMI body mass index, PS performance status, TNM tumor-node-metastasis, CAR C-reactive protein/Albumin ratio, NLR neutrophil to lymphocyte ratio, PLR platelet to lymphocyte ratio, PNI Prognostic Nutritional Index, GPS Glasgow Prognostic Score, PI prognostic index

\section{Patient demographics}

Table 1 shows the relationships between clinicopathological characteristics and survival. The median age of the patients was 68 (interquartile range [IQR], 60-75) years, and 147 patients $(75.3 \%)$ were male. The median BMI and tumor size were 21.9 (IQR 19.4-23.9) $\mathrm{kg} / \mathrm{m}^{2}$ and 60.0 (IQR, 45.0-80.0) $\mathrm{mm}$, respectively, and most patients had PS 0 (74.2\%). The median CAR, NLR, PLR, and PNI were 0.031 (IQR, 0.023-0.115), 2.06 (IQR, 1.46-2.96), 153 (IQR, 111-208), and 46.8 (IQR, 42.549.9), respectively. Most patients had GPS 0 (76.0\%) and PI 0 (91.1\%).

\section{Survival}

Survivors were followed for a median of 80 (IQR, 69124) months. Seven patients were lost to follow-up, and the shortest follow-up period for survivors was 16 months. At the time of analysis, 131 (58.2\%) patients had died. Disease recurred in 108 patients within a median duration of 14 (IQR, 7.7-26.1) months.

The 5-year OS rate for the entire study population was 48.7\%. Figure 2a-f shows Kaplan-Meier survival curves for OS according to each inflammation-based and/or nutritional marker. The five-year OS rates in the groups with low and high CAR, NLR, PLR, PNI, GPS 0 and $1 / 2$ groups, and PI 0 and $1 / 2$ groups were 50.6 and $21.4 \%$ $(p<0.001), 58.0$ and $41.2 \%(p=0.059), 53.2$ and $40.9 \%$ $(p=0.073), 39.0$ and $54.9 \%(p=0.073), 51.1$ and $41.1 \%$ $(p=0.053), 50.1$ and $35.0 \%(p=0.098)$, respectively.Among these markers, OS significantly differed only between the two CAR groups.

\section{Prognostic factors for OS}

Univariate analyses demonstrated that age, PS, location, operative procedure, TNM sub-stage, tumor size, adjuvant chemotherapy, and CAR were significantly associated with OS (Table 1). Multivariate analyses of each inflammation-based and/or nutritional marker with $p<$ 0.1 in univariate analyses revealed that high CAR (HR, 2.257; 95\% CI, 1.180-4.319; $p=0.014$ ) and high-PLR (HR, 1.478; 95\% CI, 1.025-2.133; $p=0.037$ ) were independent predictors of worse OS (Table 2).

\section{Relationship between CAR, PLR and clinicopathological variables, and CSS}

Table 3 summarizes the associations between clinicopathological variables and CAR and PLR. CAR was significantly associated with age $(p=0.004)$, PS $(p=0.006)$, 
A

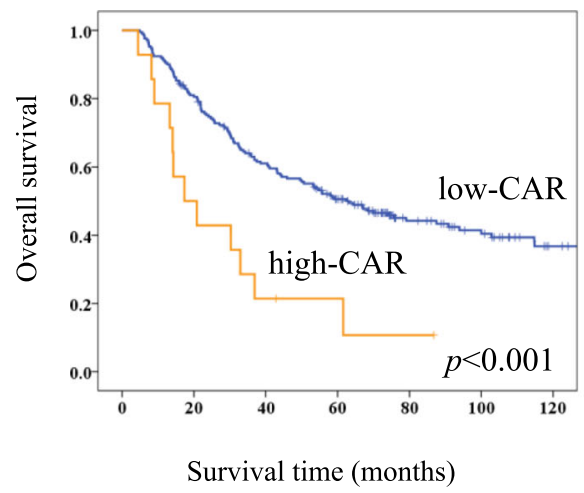

B

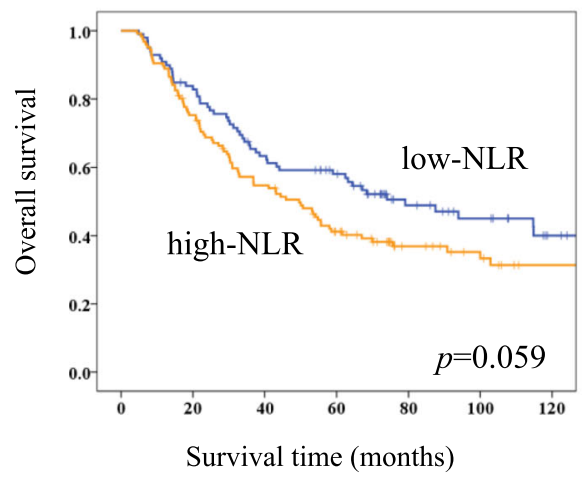

$\mathrm{C}$

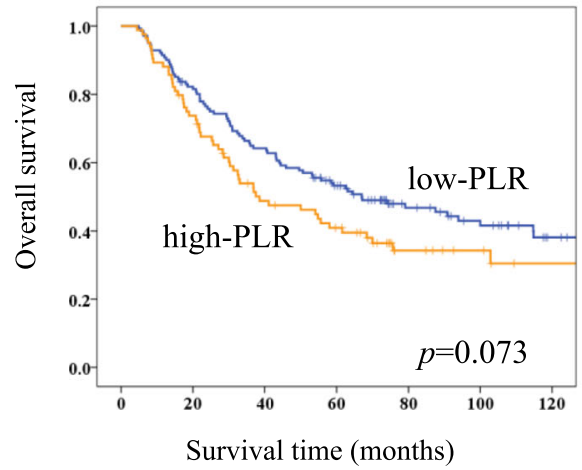

D

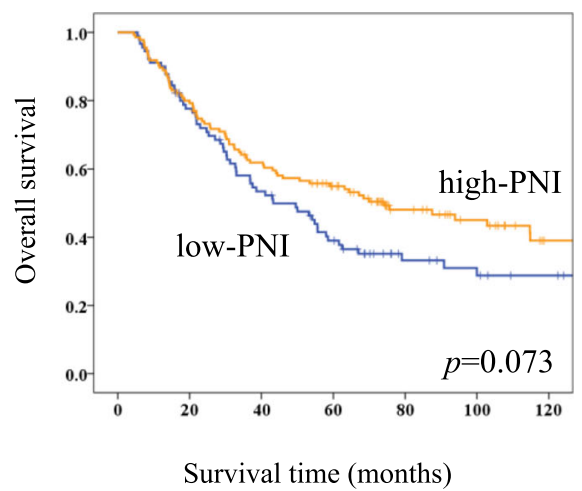

$\mathrm{E}$

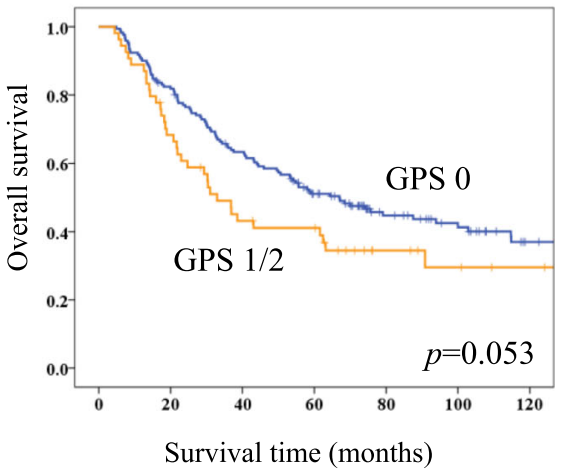

F

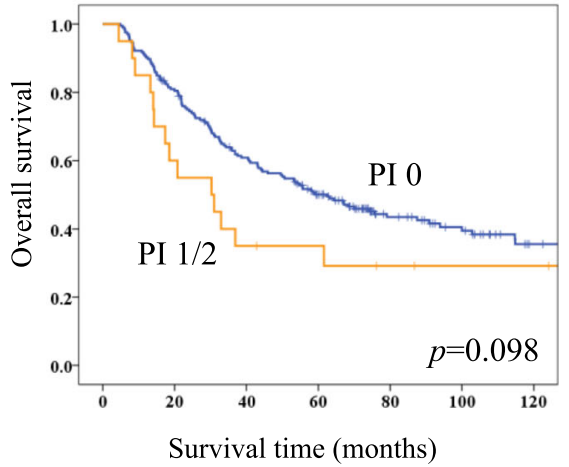

Fig. 2 Kaplan-Meier survival curves of overall survival (OS) according to inflammation-based and/or nutritional markerskaplan-Meier survival curves of OS according to CAR (a), NLR (b), PLR (c), GPS (d), PNI (e), and PI (f).

venous invasion $(0.027)$, tumor size $(p=0.027)$, and all other inflammation-based and/or nutritional markers (NLR, $p=0.004$; PLR, PNI, GPS and PI, $p<$ $0.001)$. The PLR was significantly associated with sex $(p=0.046)$, BMI $(p=0.004)$, tumor size $(p=0.001)$, and all other inflammation-based and/or nutritional markers (GPS, $p=0.011$; PI, $p=0.002$; CAR, NLR and PNI, $p<0.001)$. Figure $3 \mathrm{a}$ and $\mathrm{b}$ shows Kaplan-Meier survival curves for CSS according to CAR and PLR.
The 5-year CSS rates in the groups with low and high CAR were 57.2 and $34.3 \%(p=0.001)$, respectively, and 60.3 and $47.8 \%(p=0.036)$ in those with low and high PLR, respectively. Multivariate analyses of clinical variables with $p<0.1$ in univariate analyses and CAR and PLR revealed that high CAR (HR, 2.771; 95\% CI, 1.398-5.493; $p=0.004)$ and high PLR (HR, 1.552; 95\% CI, 1.029-2.341; $p=0.036$ ) were independent prognostic factors for CSS (Table 4). 


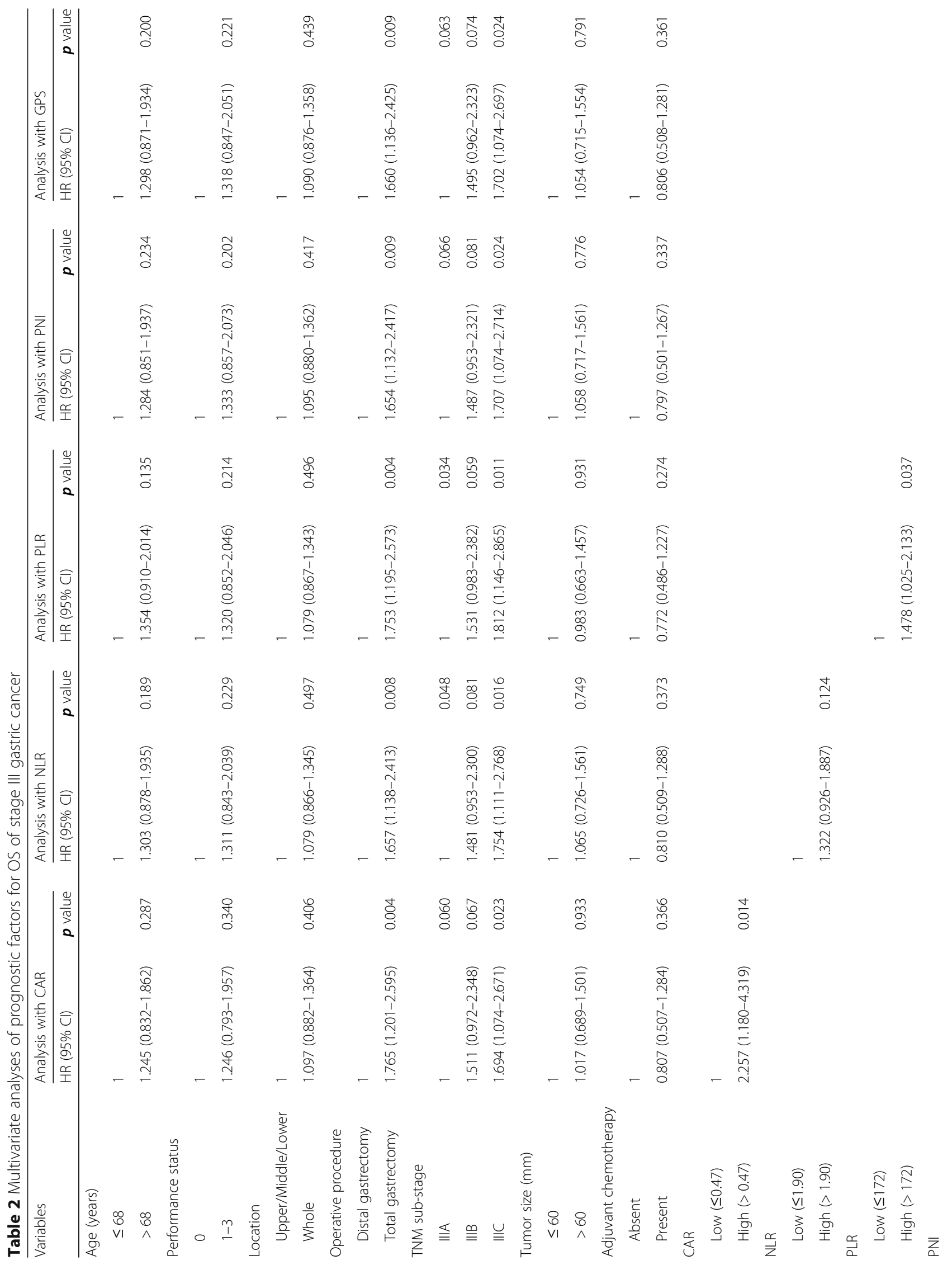




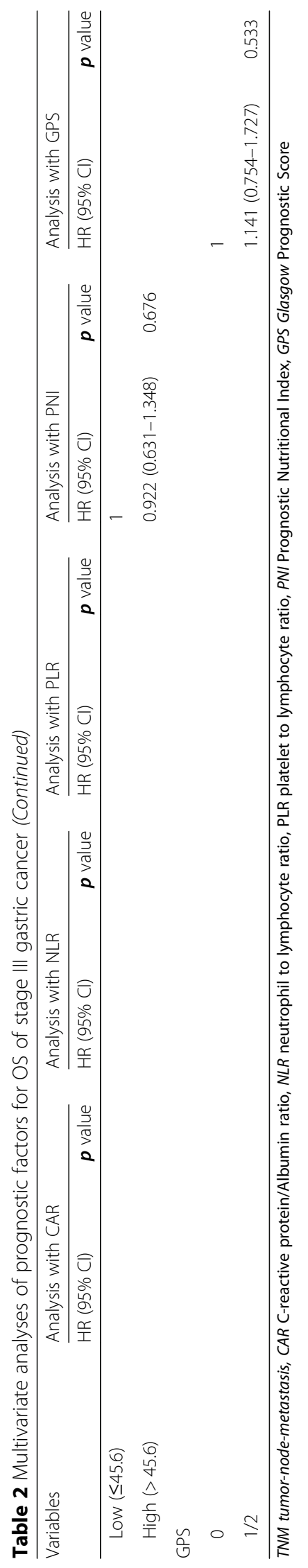


Table 3 Correlations between CAR, PLR and clinicopathological characteristics of patients

\begin{tabular}{|c|c|c|c|c|c|c|c|c|c|c|}
\hline \multirow[t]{3}{*}{ Variables } & \multicolumn{4}{|l|}{ CAR } & \multirow{3}{*}{$\begin{array}{l}p \\
\text { value }\end{array}$} & \multicolumn{4}{|l|}{$\underline{P L R}$} & \multirow{3}{*}{$\begin{array}{l}p \\
\text { value }\end{array}$} \\
\hline & \multicolumn{2}{|l|}{ Low } & \multicolumn{2}{|c|}{ High } & & \multicolumn{2}{|l|}{ Low } & \multicolumn{2}{|c|}{ High } & \\
\hline & $n$ & $\%$ & $n$ & $\%$ & & $\mathrm{n}$ & $\%$ & $n$ & $\%$ & \\
\hline \multicolumn{11}{|l|}{ Age (years) } \\
\hline$\leq 68$ & 115 & 54.5 & 2 & 14.3 & & 74 & 52.5 & 43 & 51.2 & \\
\hline$>68$ & 96 & 45.5 & 12 & 85.7 & 0.004 & 67 & 47.5 & 41 & 48.8 & 0.851 \\
\hline \multicolumn{11}{|l|}{ Sex } \\
\hline Male & 136 & 64.5 & 11 & 78.6 & & 99 & 70.2 & 48 & 57.1 & \\
\hline Female & 75 & 35.5 & 3 & 21.4 & 0.389 & 42 & 29.8 & 36 & 42.9 & 0.046 \\
\hline \multicolumn{11}{|l|}{$\mathrm{BMI}\left(\mathrm{kg} / \mathrm{m}^{2}\right)$} \\
\hline Low $(\leq 21.9)$ & 105 & 49.8 & 9 & 64.3 & & 61 & 43.3 & 53 & 63.1 & \\
\hline High (> 21.9) & 106 & 50.2 & 5 & 35.7 & 0.293 & 80 & 56.7 & 31 & 36.9 & 0.004 \\
\hline \multicolumn{11}{|l|}{ Performance status } \\
\hline 0 & 161 & 76.3 & 6 & 42.9 & & 107 & 75.9 & 60 & 71.4 & \\
\hline $1-3$ & 50 & 23.7 & 8 & 57.1 & 0.006 & 34 & 24.1 & 24 & 28.6 & 0.460 \\
\hline \multicolumn{11}{|l|}{ Location } \\
\hline U/M/L & 197 & 93.4 & 12 & 85.7 & & 132 & 93.6 & 77 & 91.7 & \\
\hline Whole & 14 & 6.6 & 2 & 14.3 & 0.261 & 9 & 6.4 & 7 & 8.3 & 0.582 \\
\hline \multicolumn{11}{|l|}{ Macroscopic type } \\
\hline Type 0/1/2 & 59 & 28.0 & 2 & 14.3 & & 41 & 29.1 & 20 & 23.8 & \\
\hline Type 3/4/5 & 152 & 72.0 & 12 & 85.7 & 0.361 & 100 & 70.9 & 64 & 76.2 & 0.390 \\
\hline \multicolumn{11}{|l|}{ Operative procedure } \\
\hline Distal gastrectomy & 110 & 52.1 & 7 & 50.0 & & 72 & 51.1 & 45 & 53.6 & \\
\hline Total gastrectomy & 101 & 47.9 & 7 & 50.0 & 0.877 & 69 & 48.9 & 39 & 46.4 & 0.716 \\
\hline \multicolumn{11}{|l|}{ Lymph node dissection } \\
\hline D1 & 30 & 14.2 & 2 & 14.3 & & 19 & 13.5 & 13 & 15.5 & \\
\hline D2 & 181 & 85.8 & 12 & 85.7 & 1.000 & 122 & 86.5 & 71 & 84.5 & 0.678 \\
\hline \multicolumn{11}{|l|}{ Histology } \\
\hline Differentiated & 91 & 43.1 & 6 & 42.9 & & 58 & 41.1 & 39 & 46.4 & \\
\hline Undifferentiated & 120 & 56.9 & 8 & 57.1 & 0.984 & 83 & 58.9 & 45 & 53.6 & 0.438 \\
\hline \multicolumn{11}{|l|}{ Lymphatic invasion } \\
\hline Absent & 18 & 8.5 & 1 & 7.1 & & 13 & 9.2 & 6 & 7.1 & \\
\hline Present & 193 & 91.5 & 13 & 92.9 & 1.000 & 128 & 90.8 & 78 & 92.9 & 0.588 \\
\hline \multicolumn{11}{|l|}{ Venous invasion } \\
\hline Absent & 150 & 71.1 & 6 & 42.9 & & 102 & 72.3 & 54 & 64.3 & \\
\hline Present & 61 & 28.9 & 8 & 57.1 & 0.027 & 39 & 27.7 & 30 & 35.7 & 0.205 \\
\hline \multicolumn{11}{|l|}{ TNM sub-stage } \\
\hline$\| \mathrm{II}$ & 77 & 36.5 & 3 & 21.4 & & 54 & 38.3 & 26 & 31.0 & \\
\hline$\| \mathrm{II}$ & 69 & 32.7 & 3 & 21.4 & & 41 & 29.1 & 31 & 36.9 & \\
\hline$\| I I C$ & 55 & 26.1 & 8 & 57.1 & 0.125 & 46 & 32.6 & 27 & 32.1 & 0.404 \\
\hline Tumor size (mm) & & & & & & & & & & \\
\hline$\leq 60$ & 112 & 53.1 & 3 & 21.4 & & 84 & 59.6 & 31 & 36.9 & \\
\hline$>60$ & 99 & 46.9 & 11 & 78.6 & 0.027 & 57 & 40.4 & 53 & 63.1 & 0.001 \\
\hline Adjuvant chemothera & & & & & & & & & & \\
\hline Absent & 37 & 17.5 & 4 & 28.6 & & 29 & 20.6 & 12 & 14.3 & \\
\hline
\end{tabular}


Table 3 Correlations between CAR, PLR and clinicopathological characteristics of patients (Continued)

\begin{tabular}{|c|c|c|c|c|c|c|c|c|c|c|}
\hline \multirow[t]{3}{*}{ Variables } & \multicolumn{4}{|l|}{ CAR } & \multirow{3}{*}{$\begin{array}{l}p \\
\text { value }\end{array}$} & \multicolumn{4}{|l|}{ PLR } & \multirow{3}{*}{$\begin{array}{l}p \\
\text { value }\end{array}$} \\
\hline & \multicolumn{2}{|l|}{ Low } & \multicolumn{2}{|c|}{ High } & & \multicolumn{2}{|l|}{ Low } & \multicolumn{2}{|c|}{ High } & \\
\hline & $n$ & $\%$ & $n$ & $\%$ & & $n$ & $\%$ & $n$ & $\%$ & \\
\hline Present & 174 & 82.5 & 10 & 71.4 & 0.292 & 112 & 79.4 & 72 & 85.7 & 0.238 \\
\hline \multicolumn{11}{|l|}{ CAR } \\
\hline Low $(\leq 0.47)$ & & & & & & 139 & 98.6 & 72 & 85.7 & \\
\hline High $(>0.47)$ & & & & & & 2 & 1.4 & 12 & 14.3 & $<0.001$ \\
\hline \multicolumn{11}{|l|}{ NLR } \\
\hline Low $(\leq 1.90)$ & 98 & 46.4 & 1 & 7.1 & & 85 & 60.3 & 14 & 16.7 & \\
\hline High (> 1.90) & 113 & 53.6 & 13 & 92.9 & 0.004 & 56 & 39.7 & 70 & 83.3 & $<0.001$ \\
\hline \multicolumn{11}{|l|}{ PLR } \\
\hline Low $(\leq 172)$ & 139 & 65.9 & 2 & 85.7 & & & & & & \\
\hline High (> 172) & 72 & 34.1 & 12 & 85.7 & $<0.001$ & & & & & \\
\hline \multicolumn{11}{|l|}{ PNI } \\
\hline Low $(\leq 45.6)$ & 78 & 37.0 & 12 & 85.7 & & 37 & 26.2 & 53 & 63.1 & \\
\hline High (> 45.6) & 133 & 63.0 & 2 & 14.3 & $<0.001$ & 104 & 73.8 & 31 & 36.9 & $<0.001$ \\
\hline \multicolumn{11}{|l|}{ GPS } \\
\hline 0 & 171 & 81.0 & 0 & 0 & & 115 & 81.6 & 56 & 66.7 & \\
\hline $1 / 2$ & 40 & 19.0 & 14 & 100 & $<0.001$ & 26 & 18.4 & 28 & 33.3 & 0.011 \\
\hline \multicolumn{11}{|l|}{ PI } \\
\hline 0 & 205 & 97.2 & 0 & 0 & & 135 & 95.7 & 70 & 83.3 & \\
\hline $1 / 2$ & 6 & 2.8 & 14 & 100 & $<0.001$ & 6 & 4.3 & 14 & 16.7 & 0.002 \\
\hline \multicolumn{11}{|l|}{ Recurrence } \\
\hline Absent & 113 & 53.6 & 4 & 28.6 & & 80 & 56.7 & 37 & 44.0 & \\
\hline Present & 98 & 46.4 & 10 & 71.4 & 0.097 & 61 & 43.3 & 47 & 56.0 & 0.065 \\
\hline
\end{tabular}

$B M I$ body mass index, PS performance status, TNM tumor-node-metastasis, CAR C-reactive protein/Albumin ratio, NLR neutrophil to lymphocyte ratio, PLR platelet to lymphocyte ratio, PNI Prognostic Nutritional Index, GPS Glasgow Prognostic Score, PI prognostic index

\section{Combined index}

According to the results of multivariate analyses, we constructed CAR-PLR score as a prognostic index, as follows: CAR-PLR score 2, both high-CAR and highPLR; CAR-PLR score 1, either high-CAR or high-PLR, but not both; and CAR-PLR score 0, neither abnormality. CAR-PLR scores were 0 for 139 patients (61.8\%), 1 for 74 patients (32.9\%), and 2 for 12 patients (5.3\%). The AUC of CAR-PLR score for predicting 5year OS was 0.573. Kaplan-Meier survival curves for OS and CSS according to CAR-PLR score are shown in Fig. 4a, b. Five-year OS and CSS rates for the CAR-PLR
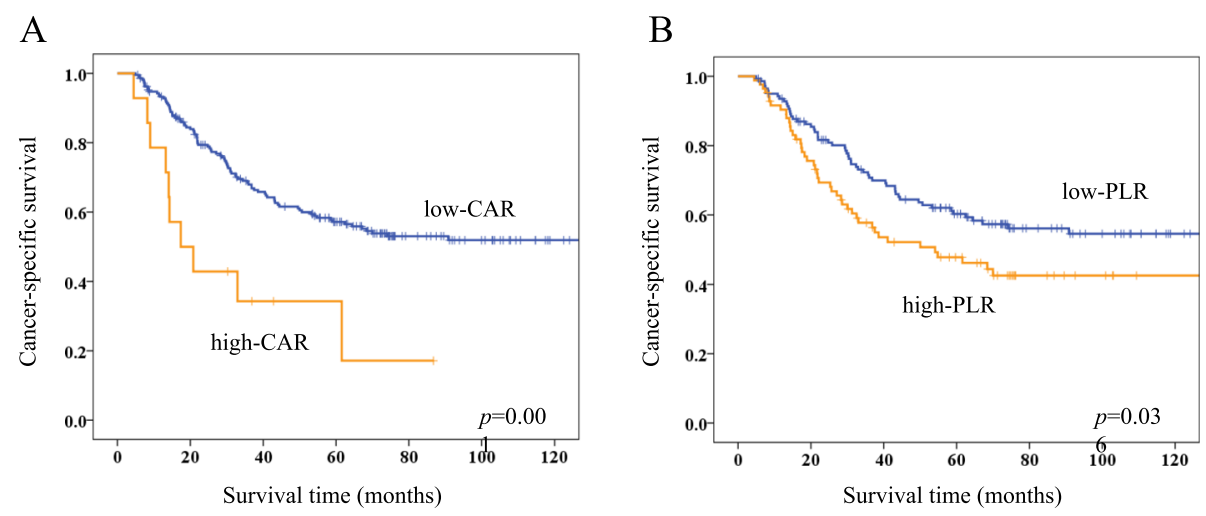

Fig. 3 Kaplan-Meier survival curves of cancer-specific survival according to CAR (a) and PLR (b) 
Table 4 Multivariate analyses of prognostic factors for CSS of stage III gastric cancer

\begin{tabular}{|c|c|c|c|c|}
\hline \multirow[t]{2}{*}{ Variables } & \multicolumn{2}{|l|}{ Analysis with CAR } & \multicolumn{2}{|l|}{ Analysis with PLR } \\
\hline & HR $(95 \%$ Cl) & $\boldsymbol{p}$ value & HR $(95 \% \mathrm{Cl})$ & $\boldsymbol{p}$ value \\
\hline \multicolumn{5}{|l|}{ Location } \\
\hline Upper/Middle/Lower & 1 & & 1 & \\
\hline Whole & 1.099 (0865-1.395) & 0.439 & $1.083(0.852-1.376)$ & 0.516 \\
\hline \multicolumn{5}{|l|}{ Operative procedure } \\
\hline Distal gastrectomy & 1 & & 1 & \\
\hline Total gastrectomy & $1.723(1.110-2.676)$ & 0.015 & $1.705(1.100-2.643)$ & 0.017 \\
\hline \multicolumn{5}{|l|}{ TNM sub-stage } \\
\hline$\| \mathrm{A}$ & 1 & 0.002 & 1 & 0.001 \\
\hline$\| \mathrm{B}$ & $2.019(1.187-3.435)$ & 0.010 & $2.015(1.184-3.432)$ & 0.010 \\
\hline$\| I I C$ & $2.617(1.534-4.467)$ & $<0.001$ & $2.799(1.635-4.792)$ & $<0.001$ \\
\hline \multicolumn{5}{|l|}{ Tumor size (mm) } \\
\hline$\leq 60$ & 1 & & 1 & \\
\hline$>60$ & $0.978(0.630-1.519)$ & 0.921 & $0.946(0.605-1.481)$ & 0.809 \\
\hline \multicolumn{5}{|l|}{ CAR } \\
\hline Low $(\leq 0.47)$ & 1 & & & \\
\hline High (>0.47) & $2.771(1.398-5.493)$ & 0.004 & & \\
\hline \multicolumn{5}{|l|}{$P L R$} \\
\hline Low $(\leq 172)$ & & & 1 & \\
\hline High (> 172) & & & $1.552(1.029-2.341)$ & 0.036 \\
\hline
\end{tabular}

score 0,1 , and 2 groups were 54.0, 42.6, and $25.0 \%(p=$ $0.006)$, and 61.2, 48.0, and $41.7 \% \quad(p=0.003)$, respectively.

\section{Discussion}

The present study evaluated and compared the prognostic values of preoperative CAR, NLR, PLR, PNI, GPS, and PI in patients with stage III gastric cancer after curative resection and verified that the results of the same analyses differed between stages II and III. We found that CAR and PLR with cut-off values of 0.47 and 172, respectively, were independent prognostic factors for OS and CSS in patients with stage III gastric cancer. These results were quite different from our previous findings in patients with stage II gastric cancer who were analyzed in the same manner [15]. To the best of our knowledge, this is the first investigation of differences in optimal inflammation-based and/or nutritional markers and optimal cut-off values as prognostic factors between stage II and III gastric cancer.

The mechanisms underlying the relationship between systemic inflammation and/or nutritional status and the
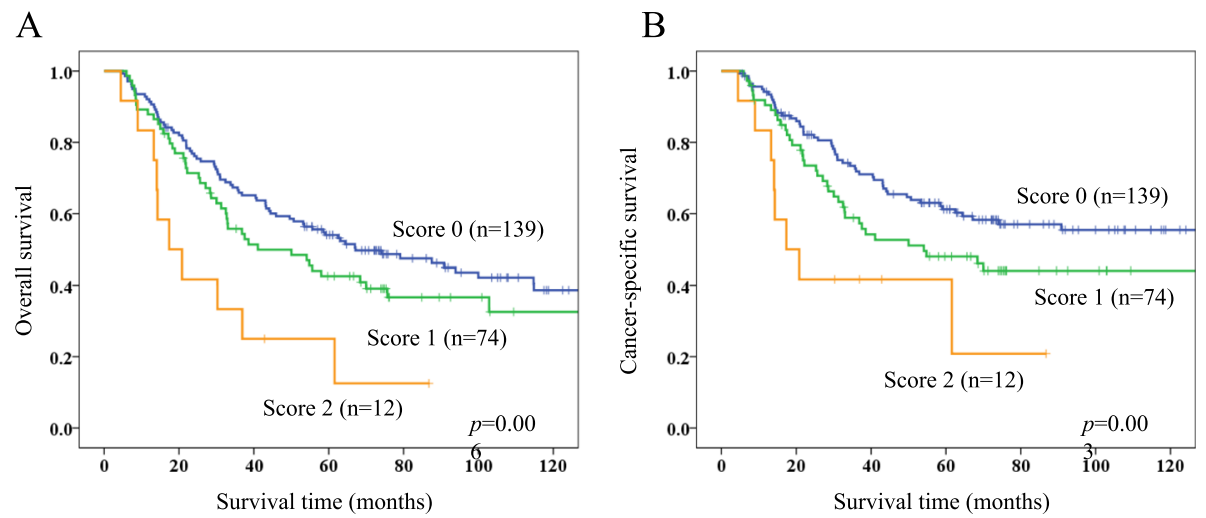

Fig. 4 Kaplan-Meier survival curves of overall survival (a) and cancer-specific survival (b) according to CAR-PLR score 
prognosis of cancer patients are complex and not yet fully elucidated. The PLR has been investigated in detail as a prognostic inflammation-based marker, and it is a prognostic factor for various types of cancer $[8$, 17]. Several studies did not find that PLR is a prognostic factor for gastric cancer [18, 19]; however, a recent meta-analysis revealed that PLR does have prognostic value for gastric cancer [20]. We identified PLR as an independent prognostic factor for patients with stage III, but not stage II cancer [15]. A higher PLR indicates relatively more platelets and fewer lymphocytes. Platelets are involved in tumor progression and metastasis. Some growth factors secreted from platelets in the tumor microenvironment, such as transforming growth factor beta (TGF- $\beta$ ), vascular endothelial growth factor (VEGF) and platelet derived growth factor (PDGF) contribute to tumor growth, angiogenesis, and tumoral neovascularization [21]. Lymphocytes play a prominent role in antitumor immunity, and lymphopenia has been associated with a worse prognosis in various cancers [22, 23]. Thus, a high PLR might have negatively affected tumor progression, which could have contributed to the poorer OS and CSS observed in the present study. Furthermore, micrometastases and residual cancer cells might be more prevalent in stage III than in stage II cancers after R0 resection. This could also explain why PLR was a prognostic factor for stage III, but not stage II gastric cancer. Consistent with our findings, a metaanalysis of 14 cohorts by $\mathrm{Gu}$ et al. concluded that elevated PLR indicated poor OS in patients with advanced, but not early stage gastric cancer [20].

We identified CAR as an independent prognostic factor in patients with stage III, as well as stage II gastric cancer, although the AUC and cutoffs were quite different for each stage. Tumor progression causes systemic inflammation and impaired nutritional status in patients with cancer. CRP is an established acute reactive protein synthesized by hepatocytes under conditions of systemic inflammation. Serum albumin is commonly used as an indicator of nutritional status. Furthermore, either serum CRP or albumin level alone are independent prognostic factors in several malignancies [24-27]. Therefore, a higher CAR might reflect a better systemic inflammatory response and worse nutritional status among cancer patients due to tumor progression and might serve as a prognostic indicator. Indeed, CAR has been indicated as an independent prognostic factor in various malignancies, including gastric cancer [3, 4]. Liu et al. reported that among NLR, PLR, GPS, and CAR, only CAR with a cut-off value of 0.025 was an independent prognostic factor in patients with gastric cancer undergoing curative resection, and a subgroup analysis according to tumor stage revealed that CAR with a cut-off value of 0.025 was significantly associated with OS in stages II and III [19]. Although CAR with a cut-off value of 0.47 was an independent prognostic factor among patients with stage III gastric cancer in the present study, the AUC of CAR with 0.510 in stage III gastric cancer was considerably lower than that of 0.641 in stage II. This finding suggests that the influence of preoperative patient-related factors represented by CAR on prognosis weakens at the more advanced stages of cancer.

We recently reported the prognostic impact of the same preoperative inflammation-based and/or nutritional markers analyzed herein in patients with stage II gastric cancer [15]. We speculated that to minimize inconsistency of results among studies, the optimal value of preoperative inflammation-based and/or nutritional markers and their cut-off values should be determined based on a specific population with a similar prognosis, which might help to make such markers reliable enough for use in clinical practice. As the appropriate method to determine cut-off values for inflammation-based and/or nutritional markers has not been established, we determined cut-off values in the present study from timedependent ROC curve analyses, which seemed to confer advantages in terms of objectivity. Furthermore, to verify our speculation, we applied the same analyses to stage II and stage III gastric cancer. Consequently, CAR and PNI with cut-off values of 0.03 and 49.2, respectively, were independent prognostic factors for OS in stage II gastric cancer, whereas CAR with a cut-off value of 0.47 and PLR with a cut-off value of 172 were independent prognostic factors in stage III. These findings supported our hypothesis that the optimal marker and cut-off values should be determined based on a specific population. On the other hand, our findings were inconsistent with those of Wang et al. [28] who found that among PNI, NLR, the lymphocyte to monocyte ratio, and PLR, only PNI was an independent prognostic factor in patients with stage III gastric cancer after curative resection, which was similar population to ours. They used median values as cut-off values for inflammation-based and/or nutritional markers, and a different method of multivariate analysis from the present study. Therefore, to reduce discrepancies among the results of various studies, a reliable and uniform method should be established to determine optimal inflammation-based and/or nutritional markers and their cut-off values to predict the prognosis of patients with cancer.

This retrospective study at a single institution has several potential limitations. The study design might have some correlation with selection bias. Our study population was relatively small because only pathological stage III gastric cancer was the study focus. Some potential cofactors affecting inflammation-based and/or nutritional markers were not controlled. Further large-scale 
prospective validation studies are needed to confirm our findings.

\section{Conclusions}

We found that CAR and PLR are independent prognostic factors for OS and CSS. These markers show more promise in terms of predicting prognosis than the established inflammation-based and/or nutritional markers, NLR, PNI, GPS, and PI in patients with stage III gastric cancer after curative resection. The optimal inflammation-based and/or nutritional markers and their cut-off values for predicting the prognosis of patients with cancer should be determined in specific populations so that they can become applicable to daily clinical practice.

\section{Abbreviations}

OS: Overall survival; CAR: C-reactive protein to albumin ratio; NLR: Neutrophils to lymphocyte ratio; PLR: Platelets to lymphocyte ratio; PNI: Prognostic nutritional index; GPS: Glasgow prognostic score; PI: Prognostic index; CRP: C-reactive protein; BMI: Body mass index; ECOG: Eastern Cooperative Oncology Group; PS: Performance status; ROC: Receiver operating characteristic; UFT: Uracil-tegafur; CT: Computed tomography; CSS: Cancer-specific survival; HR: Hazard ratio; Cl: Confidence interval; AUC: Areas under the curve; IQR: Interquartile range; TGF$\beta$ : Transforming growth factor beta; VEGF: Vascular endothelial growth factor; PDGF: Platelet derived growth factor

\section{Acknowledgements}

No acknowledgements.

\section{Authors' contributions}

TT1 and KM contributed significantly to the study design, data analysis, and drafted the manuscript. MY1, TT2, KS, NK, HT and SL participated data collection and assisted with data interpretation. MY and MO critically reviewed and revised the manuscript. All authors read and approved the final manuscript.

\section{Funding}

No funding.

\section{Availability of data and materials}

The datasets used and/or analyzed during the current study are available from the corresponding author on reasonable request.

\section{Ethics approval and consent to participate}

This retrospective study of clinical data study was approved by the ethics committee of Osaka City University (no. 3017) and was conducted in accordance with the principles of the Declaration of Helsinki.

\section{Consent for publication}

Not applicable.

\section{Competing interests}

The authors declare that they have no competing interests.

\section{Author details}

'Department of Gastroenterological Surgery, Osaka City University Graduate School of Medicine, 1-4-3 Asahimachi, Abeno-ku, Osaka 545-8585, Japan. ${ }^{2}$ Department of Gastroenterological Surgery, Osaka City General Hospital, Osaka, Japan.
Received: 17 January 2020 Accepted: 27 May 2020

Published online: 03 June 2020

\section{References}

1. Fitzmaurice C, Dicker D, Pain A, Hamavid H, Moradi-Lakeh M, Maclntyre MF, et al. The global burden of Cancer 2013. JAMA Oncol. 2015;1(4):505-27. https://doi.org/10.1001/jamaoncol.2015.0735.

2. Katai H, Ishikawa T, Akazawa K, Isobe Y, Miyashiro I, Oda I, et al. Five-year survival analysis of surgically resected gastric cancer cases in Japan: a retrospective analysis of more than 100,000 patients from the nationwide registry of the Japanese gastric Cancer association (2001-2007). Gastric Cancer. 2018;21(1):144-54. https://doi.org/10.1007/s10120-017-0716-7.

3. Deng TB, Zhang J, Zhou YZ, Li WM. The prognostic value of C-reactive protein to albumin ratio in patients with lung cancer. Medicine. 2018;97(50): e13505. https://doi.org/10.1097/MD.0000000000013505.

4. Kudou K, Saeki H, Nakashima Y, Kamori T, Kawazoe T, Haruta Y, et al. Creactive protein/albumin ratio is a poor prognostic factor of esophagogastric junction and upper gastric cancer. J Gastroenterol Hepatol. 2019;34(2):355-63. https://doi.org/10.1111/jgh.14442.

5. Wang SC, Chou JF, Strong VE, Brennan MF, Capanu M, Coit DG Pretreatment neutrophil to lymphocyte ratio independently predicts disease-specific survival in Resectable Gastroesophageal junction and gastric adenocarcinoma. Ann Surg. 2016;263(2):292-7. https://doi.org/10.1097/SLA. 0000000000001189.

6. Zhang Y, Lu JJ, Du YP, Feng CX, Wang LQ, Chen MB. Prognostic value of neutrophil-to-lymphocyte ratio and platelet-to-lymphocyte ratio in gastric cancer. Medicine. 2018;97(12):e0144. https://doi.org/10.1097/MD. 0000000000010144

7. Yodying H, Matsuda A, Miyashita M, Matsumoto S, Sakurazawa N, Yamada $M$, et al. Prognostic significance of neutrophil-to-lymphocyte ratio and platelet-to-lymphocyte ratio in oncologic outcomes of esophageal Cancer: a systematic review and meta-analysis. Ann Surg Oncol. 2016;23(2):646-54. https://doi.org/10.1245/s10434-015-4869-5.

8. Yu J, Ding Z, Yang Y, Liu S. Increased platelet-to-lymphocytes ratio is associated with poor long-term prognosis in patients with pancreatic cancer after surgery. Medicine. 2018;97(25):e11002. https://doi.org/10.1097/ MD.0000000000011002.

9. Okada S, Shimada J, Kato D, Tsunezuka H, Teramukai S, Inoue M. Clinical significance of prognostic nutritional index after surgical treatment in lung Cancer. Ann Thorac Surg. 2017;104(1):296-302. https://doi.org/10.1016/j. athoracsur.2017.01.085

10. Luo Z, Zhou L, Balde Al, Li Z, He L, ZhenWei C, et al. Prognostic impact of preoperative prognostic nutritional index in resected advanced gastric cancer: a multicenter propensity score analysis. Eur J Surg Oncol. 2019;45(3): 425-31. https://doi.org/10.1016/j.ejso.2018.09.004.

11. Yamada S, Fujii T, Yabusaki N, Murotani K, Iwata N, Kanda M, et al. Clinical implication of inflammation-based prognostic score in pancreatic Cancer: Glasgow prognostic score is the Most reliable parameter. Medicine. 2016: 95(18):e3582. https://doi.org/10.1097/MD.0000000000003582.

12. Melling $N$, Gruning $A$, Tachezy M, Nentwich $M$, Reeh M, Uzunoglu FG, et al. Glasgow prognostic score may be a prognostic index for overall and perioperative survival in gastric cancer without perioperative treatment. Surgery. 2016;159(6):1548-56. https://doi.org/10.1016/j.surg.2016.01.018.

13. Proctor MJ, Morrison DS, Talwar D, Balmer SM, Fletcher CD, O'Reilly DS, et al A comparison of inflammation-based prognostic scores in patients with cancer. A Glasgow inflammation outcome study. Eur J Cancer. 2011;47(17): 2633-41. https://doi.org/10.1016/j.ejca.2011.03.028.

14. Pan QX, Su ZJ, Zhang JH, Wang CR, Ke SY. A comparison of the prognostic value of preoperative inflammation-based scores and TNM stage in patients with gastric cancer. Onco Targets Ther. 2015;8:1375-85. https://doi.org/10. 2147/OTT.S82437.

15. Toyokawa T, Muguruma K, Tamura T, Sakurai K, Amano R, Kubo N, et al. Comparison of the prognostic impact and combination of preoperative inflammation-based and/or nutritional markers in patients with stage II gastric cancer. Oncotarget. 2018;9(50):29351-64. https://doi.org/10.18632/ oncotarget.25486.

16. Japanese Gastric Cancer Association. Japanese classification of gastric carcinoma: 3rd English edition. Gastric Cancer. 2011;14(2):101-12.

17. Ma JY, Ke LC, Liu Q. The pretreatment platelet-to-lymphocyte ratio predicts clinical outcomes in patients with cervical cancer: a meta-analysis. Medicine. 2018;97(43):e12897. https://doi.org/10.1097/MD.0000000000012897. 
18. Kim EY, Lee JW, Yoo HM, Park CH, Song KY. The platelet-to-lymphocyte ratio versus neutrophil-to-lymphocyte ratio: which is better as a prognostic factor in gastric Cancer? Ann Surg Oncol. 2015;22(13):4363-70. https://doi.org/10. 1245/s10434-015-4518-z.

19. Liu X, Sun X, Liu J, Kong P, Chen S, Zhan Y, et al. Preoperative C-reactive protein/albumin ratio predicts prognosis of patients after curative resection for gastric Cancer. Transl Oncol. 2015;8(4):339-45. https://doi.org/10.1016/j. tranon.2015.06.006.

20. Gu X, Gao XS, Cui M, Xie M, Peng C, Bai Y, et al. Clinicopathological and prognostic significance of platelet to lymphocyte ratio in patients with gastric cancer. Oncotarget. 2016;7(31):49878-87. https://doi.org/10.18632/ oncotarget.10490.

21. Palacios-Acedo AL, Mege D, Crescence L, Dignat-George F, Dubois C, Panicot-Dubois L. Platelets, Thrombo-inflammation, and Cancer: collaborating with the enemy. Front Immunol. 2019;10:1805. https://doi.org/ 10.3389/fimmu.2019.01805

22. Kobayashi N, Usui S, Kikuchi S, Goto Y, Sakai M, Onizuka M, et al. Preoperative lymphocyte count is an independent prognostic factor in node-negative non-small cell lung cancer. Lung Cancer. 2012;75(2):223-7. https://doi.org/10.1016/j.lungcan.2011.06.009.

23. Feng JF, Liu JS, Huang Y. Lymphopenia predicts poor prognosis in patients with esophageal squamous cell carcinoma. Medicine. 2014;93(27):e257. https://doi.org/10.1097/MD.0000000000000257.

24. Kersten C, Louhimo J, Algars A, Lahdesmaki A, Cvancerova M, Stenstedt K, et al. Increased C-reactive protein implies a poorer stage-specific prognosis in colon cancer. Acta Oncol. 2013;52(8):1691-8. https://doi.org/10.3109/ 0284186X.2013.835494.

25. Yu Q, Yu XF, Zhang SD, Wang HH, Wang HY, Teng LS. Prognostic role of Creactive protein in gastric cancer: a meta-analysis. Asian Pac J Cancer Prev. 2013;14(10):5735-40. https://doi.org/10.7314/apjcp.2013.14.10.5735.

26. Onate-Ocana LF, Aiello-Crocifoglio V, Gallardo-Rincon D, Herrera-Goepfert R, Brom-Valladares R, Carrillo JF, et al. Serum albumin as a significant prognostic factor for patients with gastric carcinoma. Ann Surg Oncol. 2007; 14(2):381-9. https://doi.org/10.1245/s10434-006-9093-x.

27. Chen Z, Shao Y, Wang K, Cao W, Xiong Y, Wu R, et al. Prognostic role of pretreatment serum albumin in renal cell carcinoma: a systematic review and meta-analysis. Onco Targets Ther. 2016;9:6701-10. https://doi.org/10 2147/OTT.S108469

28. Wang HX, Wang CC, Yang W, Gao LL, Yu SQ. Prognostic value of preoperative prognostic nutritional index in stage III gastric cancer after curative resection: a retrospective cohort study. Asia Pac J Clin Nutr. 2018; 27(3):540-5. https://doi.org/10.6133/apjcn.072017.03.

\section{Publisher's Note}

Springer Nature remains neutral with regard to jurisdictional claims in published maps and institutional affiliations.

Ready to submit your research? Choose BMC and benefit from:

- fast, convenient online submission

- thorough peer review by experienced researchers in your field

- rapid publication on acceptance

- support for research data, including large and complex data types

- gold Open Access which fosters wider collaboration and increased citations

- maximum visibility for your research: over $100 \mathrm{M}$ website views per year

At $\mathrm{BMC}$, research is always in progress.

Learn more biomedcentral.com/submissions 